\title{
Comparative efficacy and tolerability of front-line treatments for newly diagnosed chronic-phase chronic myeloid leukemia: an update network meta-analysis
}

Lu Tang ${ }^{1,2}$, Huan Zhang ${ }^{4}$, Yi-zhong Peng ${ }^{5}$, Cheng-gong Li $i^{1,2}$, Hui-wen Jiang ${ }^{1,2}$, Min Xu' ${ }^{1}$, Heng Mei $i^{1,2,3^{*}}$ (D) and $\mathrm{Yu} \mathrm{Hu} u^{1,2,3^{*}}$

\begin{abstract}
Background: Recent years have witnessed the rapid evolution of therapies in chronic-phase chronic myeloid leukemia (CP-CML). To assess the efficacy and tolerability of all reported front-line treatments for patients with newly diagnosed CML, a multiple-treatments meta-analysis was performed, which accounted for both direct and indirect comparisons among those treatments.

Methods: Primary outcomes were the percentage of patients achieving major molecular response (MMR) and complete cytogenetic response (CCyR) within 12 months. Secondary outcomes included the percentage of progression to accelerated phase (AP), serious adverse effects (AEs), overall discontinuation and discontinuation for drug-related AEs. Direct pairwise meta-analysis and indirect multi-comparison meta-analysis among those treatments in each outcome were both conducted. The surface under the cumulative ranking curve (SUCRA) was calculated for all treatments in each outcome. Cluster analysis demonstrated the division of treatments into distinct groupings according to efficacy and tolerability profiles.

Results: A total of 21 randomized controlled trials (RCTs, including 10,187 patients) comparing 15 different interventions for CP-CML patients were included in this study. SUCRA analysis suggested that all tyrosine kinase inhibitors (TKIs) are highly effective in newly diagnosed CP-CML when compared to traditional drugs. Newer TKls and higher-dose imatinib generally resulted in faster cytogenetic and molecular responses when compared with standard-dose imatinib and traditional drugs. Furthermore, traditional drugs, higher-dose imatinib and newer TKls demonstrated lower acceptability than standard-dose imatinib. One cluster of interventions, which included nilotinib (300/400 mg BID), dasatinib (100 mg QD) and radotinib (300 mg BID), demonstrated higher efficacy and tolerability than other treatments.

Conclusions: Nilotinib (300/400 mg BID), dasatinib (100 mg QD) and radotinib (300 mg BID) prove to be the most recommended front-line treatments of the greatest efficacy and tolerability for CP-CML patients. High-dose therapies are recommended only for patients in accelerated phase/blast phase or with suboptimal CML-CP response, and management of adverse events should be carried out to avoid compromising the clinical efficacy.
\end{abstract}

Keywords: Chronic myeloid leukemia, Network meta-analysis, Efficacy, Tolerability, Tyrosine kinase inhibitors

\footnotetext{
* Correspondence: hmei@hust.edu.cn; dr_huyu@126.com

${ }^{1}$ Institute of Hematology, Union Hospital, Tongji Medical College, Huazhong

University of Science and Technology, 1277 Jiefang Road, Wuhan 430022,"

Hubei, China

Full list of author information is available at the end of the article
}

(c) The Author(s). 2019 Open Access This article is distributed under the terms of the Creative Commons Attribution 4.0 International License (http://creativecommons.org/licenses/by/4.0/), which permits unrestricted use, distribution, and reproduction in any medium, provided you give appropriate credit to the original author(s) and the source, provide a link to the Creative Commons license, and indicate if changes were made. The Creative Commons Public Domain Dedication waiver (http://creativecommons.org/publicdomain/zero/1.0/) applies to the data made available in this article, unless otherwise stated. 


\section{Background}

Chronic myeloid leukemia (CML) is one specific category of myeloproliferative neoplasm (MPN), characterized by an excessive proliferation of moderately and well differentiated cells of the granulocytic lineage [1]. The molecular abnormity of CML is the presence of an abnormal Philadelphia (Ph) chromosome, formed by a reciprocal translocation between the long arms of chromosomes 9 (ch9) and 22 (ch22). Central pathogenesis of CML is the fusion of the Abelson murine leukemia (ABL1) gene on ch9 with the breakpoint cluster region (BCR) gene on ch22, which results in expression of an oncoprotein termed BCR/ABL1 [2]. Compared to wild-type C-ABL1, BCR/ABL1 fusion protein displays increased kinase activity, which makes it a necessary and sufficient initiating trigger in CML [3]. According to conservative statistics, CML accounts for approximately $15 \%$ of adult leukemia, with an annual incidence of 1-2 cases per 100,000 persons. The diagnostic criteria, clinical characteristics, and natural course of the disease have been well defined in recent evidencebased guidelines for the diagnosis and management of CML [4]. The majority of diagnoses are made in the chronic phase (CP-CML) as opposed to the accelerated phase (AP-CML), therefore it is of great importance to confirm best front-line treatments in newly diagnosed CP-CML.

Before 2000, while the allogeneic stem cell transplant (Allo-SCT) offered greater chance of long-term survival, the mainstay of treatment for individuals ineligible for transplant was limited to interferon-alfa (IFN- $\alpha$ ), busulfan, hydroxyurea $(\mathrm{Hu})$ or chemotherapy $[3,5]$. IFN- $\alpha$ led to disease regression and improved survival but was hindered by its limited efficacy and associated significant toxicities. Allo-SCT is curative, but carries great risks of mortality. In recent years, the CML therapeutic landscape has changed dramatically with the development of the small molecule tyrosine kinase inhibitors (TKIs) that potently interfered with the interaction between the BCR/ABL1 oncoprotein and adenosine triphosphate (ATP), blocking cellular proliferation of the malignant clone. This "targeted" approach altered the natural history of CML, improving the 10-year survival rate from approximately 20 to $80 \%-90 \%$ [6].

The previous systematic reviews and meta-analyses performed a direct comparison of the relative efficacy of two or more kinds of tyrosine kinase inhibitors for newly diagnosed CP-CML [7, 8]. Hofmann's meta-analysis compared the major molecular response during the first year of standard-dose imatinib and high-dose imatinib or second-generation TKIs for chronic myeloid leukemia [9]. Yun' study compared the outcomes of new generation TKIs versus imatinib in patients with newly diagnosed CP-CML, and concluded that new generation
TKIs resulted in a greater major molecular response [10]. Chen' group [11] conducted a network meta-analysis (NMA) of first-line treatments for CP-CML, and Fachi' study [12] performed a NMA to compare the efficacy and safety of several TKIs. Although the previous studies conducted direct or indirect comparison among different therapies in CP-CML, none of them made a comprehensive comparison of all reported treatments, including conventional drugs, imatinib and new TKIs. Additionally, the dose difference of each drug may result in variation in efficacy. More importantly, the relative risks of serious adverse effect and treatment discontinuation should also be taken into consideration when we evaluate each kind of therapy. Herein, our study was the first meta-analysis that was based on multiple treatments to simultaneously assess the comparative efficacy and tolerability of almost all front-line treatments for newly diagnosed CML patients.

\section{Methods}

This multiple comparison NMA was conducted in accordance with the recommendations of the Cochrane Comparing Multiple Interventions Methods Group [13] and the Preferred Reporting Items for Systematic Reviews and Meta-Analyses (PRISMA) extension statement for systematic reviews incorporating NMAs [14].

\section{Literature search}

Two authors (Tang and Mei) independently used the following tools: MEDLINE, EMBASE, Cochrane library databases and ClinicalTrials.gov website to obtain relevant articles published until now. Following the PICOS principle (Participants, Interventions, Comparisons, Outcomes and Study design), the key search terms included "chronic myeloid leukemia, treatment, efficacy, safety, imatinib, nilotinib, bosutinib, dasatinib, radotinib, ponatinib, interferon, cytarabine, chemotherapy". The complete search used for PubMed was: $((()((()((($ chemotherapy [Title/Abstract]) OR cytarabine [Title/Abstract]) OR interferon [Title/Abstract]) OR ponatinib [Title/Abstract]) OR radotinib [Title/Abstract]) OR dasatinib [Title/Abstract]) OR bosutinib [Title/Abstract]) OR nilotinib [Title/ Abstract]) OR imatinib [Title/Abstract]) OR treatment [Title/Abstract])) AND ((tolerability [Title/Abstract]) OR (efficacy [Title/Abstract])) AND chronic myeloid leukemia [Title/Abstract] Sort by: Best Match Filters: Clinical Trial; Humans. All eligible studies were considered for this review, and we also did a manual search, using the reference lists of key articles published.

\section{Outcome measures and eligibility criteria}

Primary outcomes were the percentage of patients achieving major molecular response (MMR) and complete cytogenetic response (CCyR) within 12 
months. Secondary outcomes included the percentage of progression to accelerated phase (AP), serious adverse effects (AEs in 3 or 4 grade), overall discontinuation and discontinuation for drug-related AEs. MMR is defined as achieving a ratio of $\mathrm{BCR}-\mathrm{ABL} 1 \leq 0.1 \%$ on the international scale $(\leq 0.1 \%$ BCR-ABL1[IS]) measured by reverse transcription-quantitative polymerase chain reaction ( $\mathrm{RT}-\mathrm{qPCR}$ ) or $\geq 3$-log reduction in $\mathrm{BCR}-\mathrm{ABL} 1$ mRNA from the standard baseline if RT-qPCR is not available [15]. CCyR is defined as achieving 0\% Philadelphia chromosome-positive $(\mathrm{Ph}+)$ metaphases by cytogenetic analysis of bone marrow [16]. Two researchers (Tang and Mei) independently assessed all the included studies and extracted the data. Studies were considered eligible if they met all the following inclusion criteria: (1) randomized controlled trials (RCTs) comparing at least two treatments as first line treatment for newly diagnosed, previously untreated (except for treatment with hydroxyurea or anagrelide) CP-CML patients; (2) the diagnosis of CML according to the trials was based on cytogenetic, fluorescence in situ hybridization (FISH) and/or RT-qPCR results; (3) sample size $\geq 40$; (4) sufficient follow-up data about the above outcomes. When there were several reports concerning the same study, we included the high quality and most recent publication in our meta-analysis. Disagreements between the two reviewers were resolved by discussion with another reviewer $(\mathrm{Hu})$.

\section{Assessment of risk of bias}

As for quality assessment, the following domains were taken into consideration: random sequence generation, allocation concealment, blinding (self-reported), blinding (objective outcomes), incomplete and selective outcome reporting, and other bias presence. We made critical assessment separately for each domain and graded it as low risk for bias, unclear risk, or high risk for bias according to the criteria specified in the Cochrane Handbook [17].

\section{Data extraction}

Data extraction was independently performed by two researchers (Tang and Mei), and any disagreement was resolved by a third researcher $(\mathrm{Hu})$. For each $\mathrm{RCT}$, the following characteristics were collected: the first author; publication year; trial number; study design, number of patients in each arm; interventions, gender and age distribution in participants, CML scoring systems (including Sokal risk and Hasford risk), ECOG (Eastern Cooperative Oncology Group) performances status and any relevant outcomes in this meta-analysis.

\section{Data synthesis and analysis}

We produced visual inspection of separate network diagrams to show the amount of evidence available for each outcome in STATA v15.0. In each network plot, the size of each node is proportional to the total number of randomized participants (sample size) allocated to the corresponding treatment across all trials, and the width of each line is proportional to the total number of RCTs evaluating the corresponding treatment comparison. Odds ratios (ORs) with corresponding 95\% confidence intervals (95\% CIs) were calculated for dichotomous outcomes.

First, the pairwise meta-analysis was conducted to compare the same interventions to incorporate the assumption that the different studies were estimating different, yet related, treatment effects. Statistical heterogeneity was examined using the Cochran's Q-statistic and a P-value of less than 0.01 was considered significant. $\mathrm{I}^{2}$ test was also used to quantify heterogeneity (ranging from 0 to100\%). $P<0.01$ for $\mathrm{Q}$-test or $\mathrm{I}^{2}>50 \%$ indicated the existence of heterogeneity across the studies. To minimizes the effect of heterogeneity, random-effect model was used. All statistical analysis in traditional meta-analysis was conducted using STATA v15.0.

Additionally, we made inferences between two intervention arms, such as $A$ versus $B$, from indirect evidence (from combining studies through another intermediate comparator C) [18]. Network Meta-Analysis (NMA) is a technique to meta-analyze more than two interventions at the same time. Using a full Bayesian evidence network, all indirect comparisons are conducted to arrive at a single, integrated, estimate of the effect of all included treatments based on all included studies. Thus, even if there are no known comparisons for the investigated intervention, a network meta-analysis still can estimate the potential effect of this intervention based on existing head-to-head trials. We performed this network metaanalysis with a random-effects model based on a Bayesian framework using Markov Chain Monte Carlo methods in WinBUGS and R v3.0.2. To rank the treatments based on efficacy and safety, a probabilistic analysis was performed to estimate rank probabilities based on NMA, and the rank probabilities were summarized for each intervention in order to obtain the surface under the cumulative ranking curve (SUCRA). SUCRA analysis could illustrate the outcome percentages of every treatment relative to an ideal treatment, which always ranks first without uncertainty. The inconsistency refers to disagreements between direct and indirect evidence [19], and was estimated by the node-splitting method which generates $P$ values for the null hypothesis that there is no significant inconsistency between direct and indirect evidence [20]. In case of significant inconsistency, we investigated the distribution of clinical and methodological variables that we suspected might be potential sources of either heterogeneity or inconsistency in every comparison-specific group of trials. 
Finally, we produced a clustered ranking plot including SUCRA value for efficacy on the $\mathrm{x}$-axis and SUCRA value for tolerability on the $y$-axis. Cluster analysis demonstrated the division of treatments into distinct groupings according to efficacy and tolerability profiles.

\section{Result}

\section{Study characteristics and risk of bias assessment}

A total of 2231 records were identified through the primary search, combined with additional 165 studies searched through ClinicalTrials.gov website (Fig. 1). Within these 2396 references, 741 were identified as ineligible due to duplication, leaving 1655 studies for selection, of which 1614 proved ineligible on the basis of titles, abstracts and full-text screening, leaving 34 eligible studies. 21 RCTs from 34 articles [21-54] involving 10, 187 newly diagnosed CP-CML patients were included in this network meta-analysis. The characteristics of the included trials are summarized and presented in Table 1 and Additional file 1: Table S1. 20 trials (95.24\%) described an adequate random sequence generation, and adequate treatment allocation concealment in 18 trials (85.71\%). Double-blind (patients and treatment executors) strategies were carefully performed in 15 trials (71.42\%), and blind strategies for objectively outcome assessors were involved in 20 trials $(95.24 \%)$. The detailed assessment of the risk of bias is provided in Additional file 1: Table S2 and Additional file 2: Figure S1.

\section{Network geometry}

The six network graphical structures for each outcome display the available direct comparisons of the network of trials organized from the included RCTs (Fig. 2). The recommended standard treatment "imatinb $400 \mathrm{mg}$ QD" were thoroughly compared against every other treatment. Novel drug, such as ponatinib, radotinib and bosutinib were only compared against standard treatment "imatinib 400mg QD". MMR and CCyR within 12 months (Fig. 2a-b) were reported in almost all trials (21 RCTs including 10,187 patients and 19 RCTs including 9673 patients, respectively), progression to AP-CML and serous AEsc (Fig. 2c-d) were reported in quite limited trials (8 RCTs including 5712 patients and 10 RCTs including 4152 patients, respectively), whereas overall discontinuation and discontinuation for drug-related AEs (Fig. 2e-f) were both reported in 18 trials (8209 and 7411 patients, respectively).

\section{Direct pairwise meta-analysis}

All treatments had at least one comparison with the standard treatment "imatinib $400 \mathrm{mg}$ QD", and several of them were directly compared with two or more other treatments (Additional file 1: Table S3). As for efficacy,

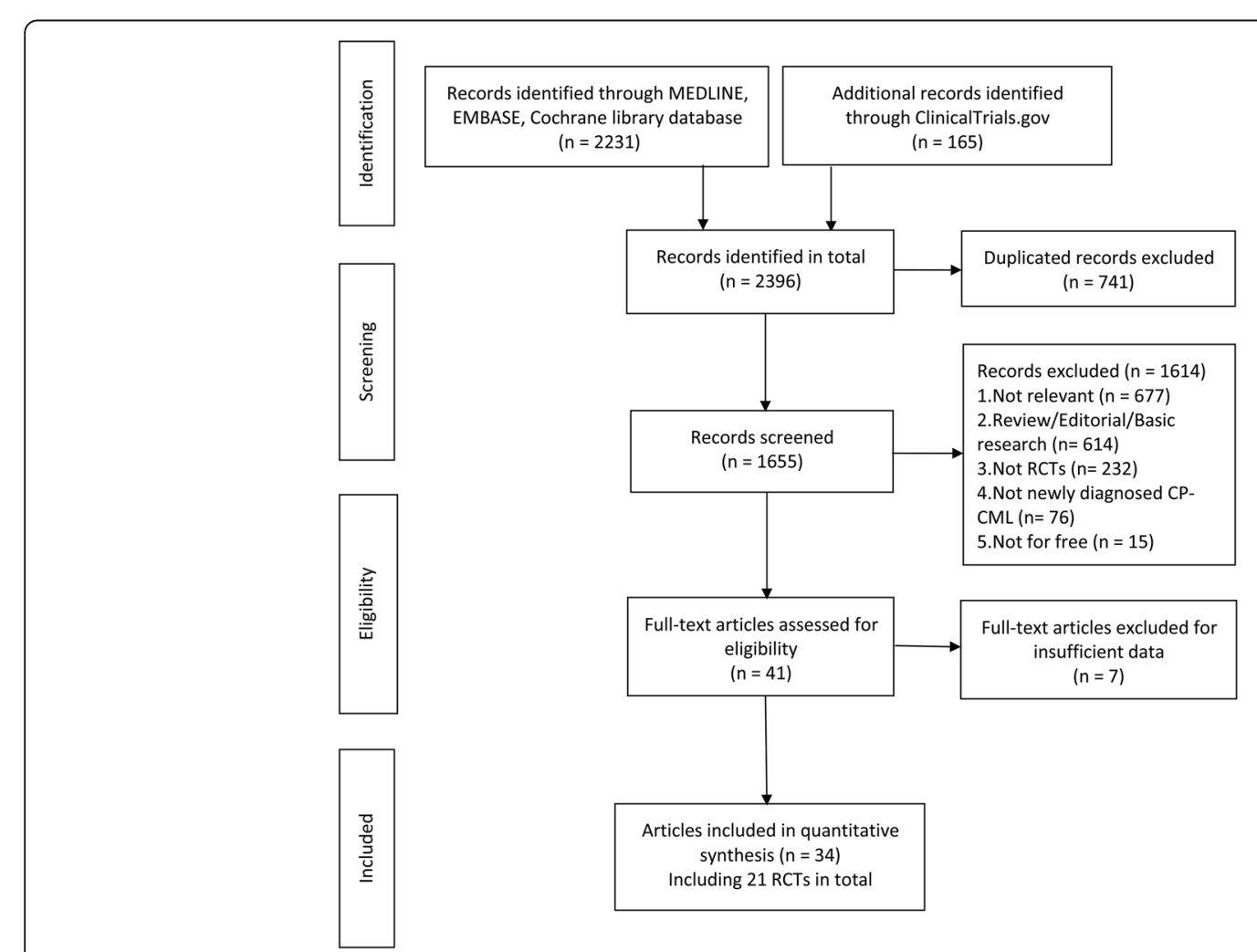

Fig. 1 Flow diagram of selecting relevant published RCTs regarding front-line treatments in newly diagnosed CP-CML 


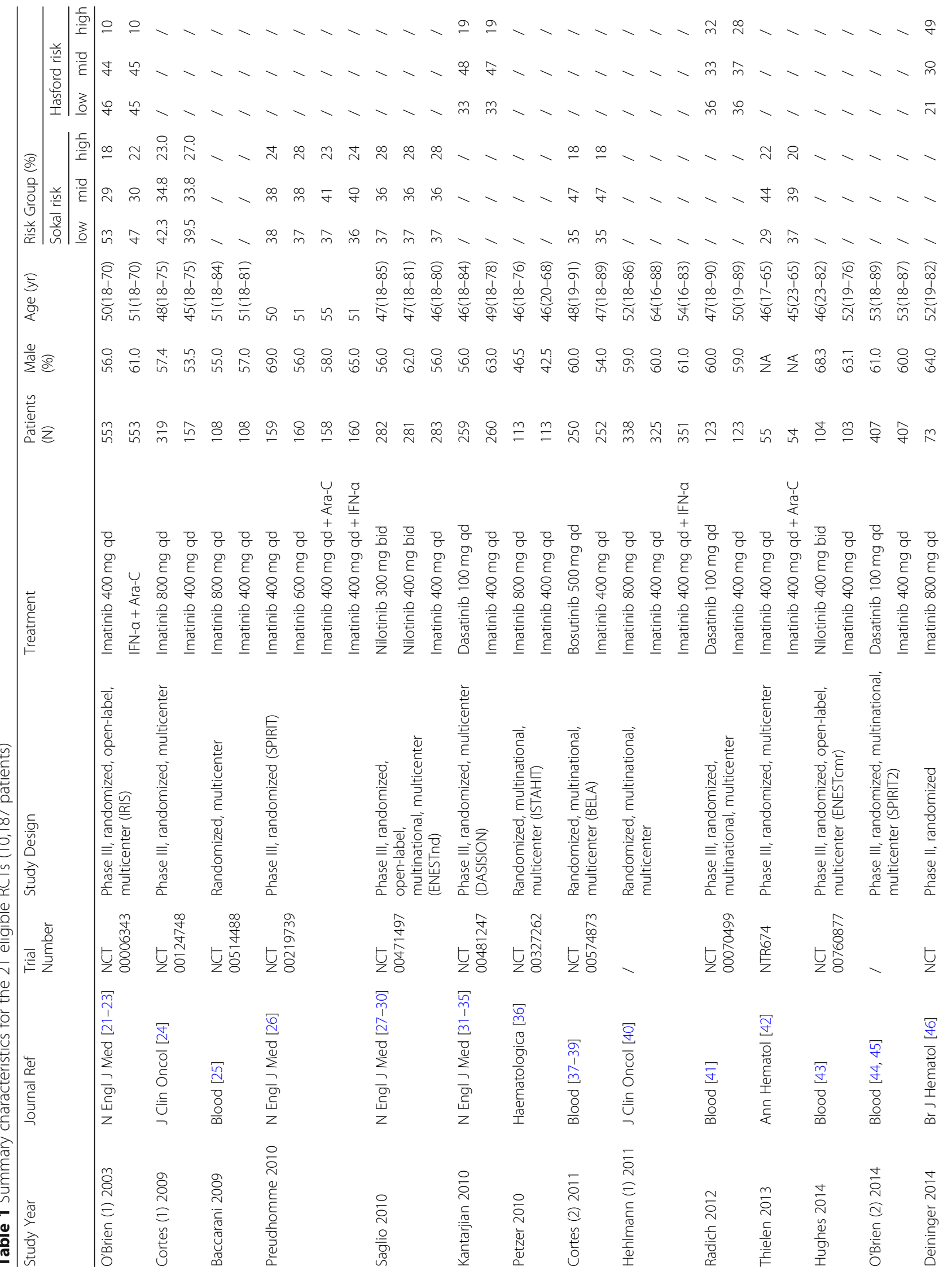




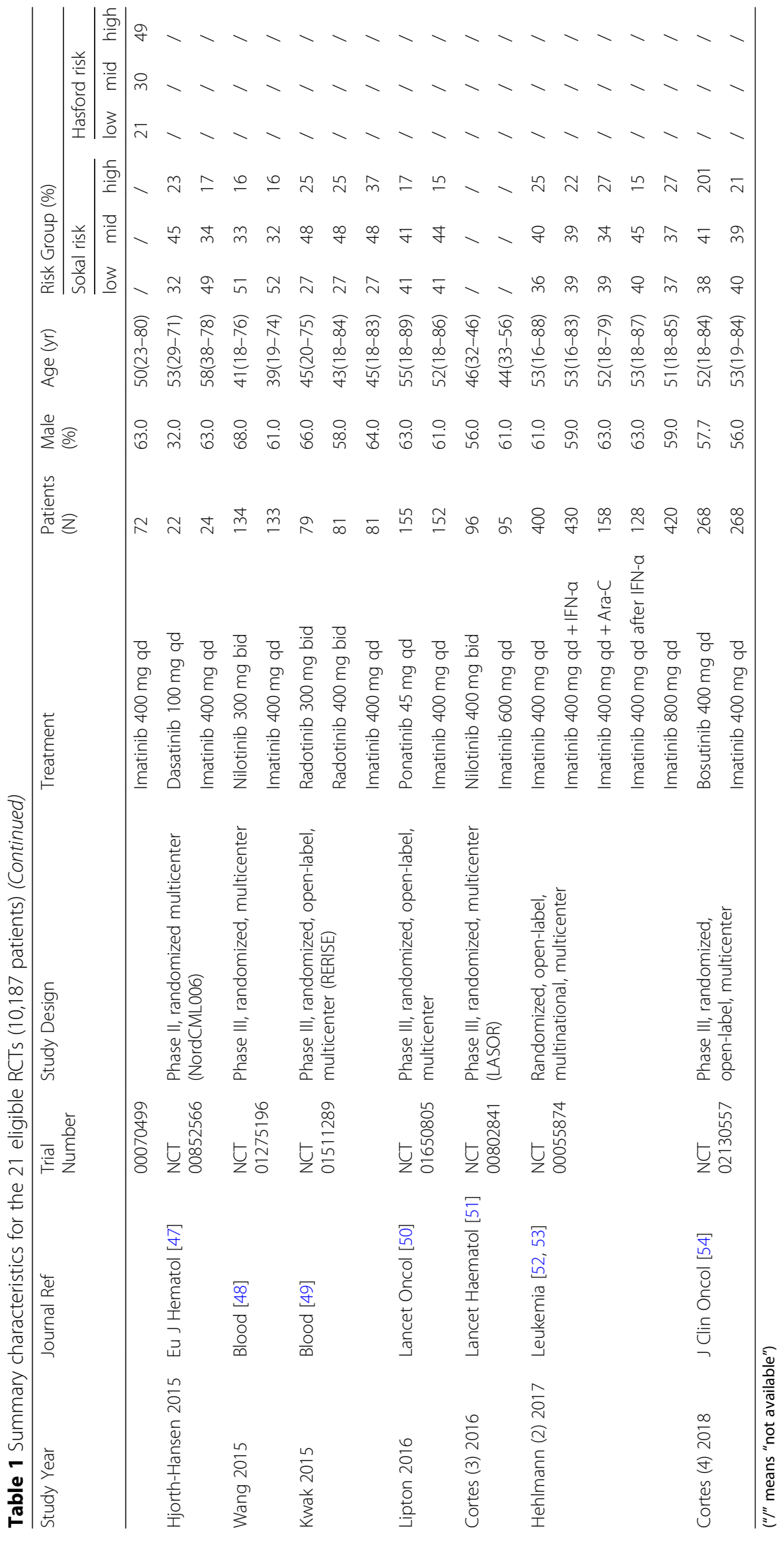




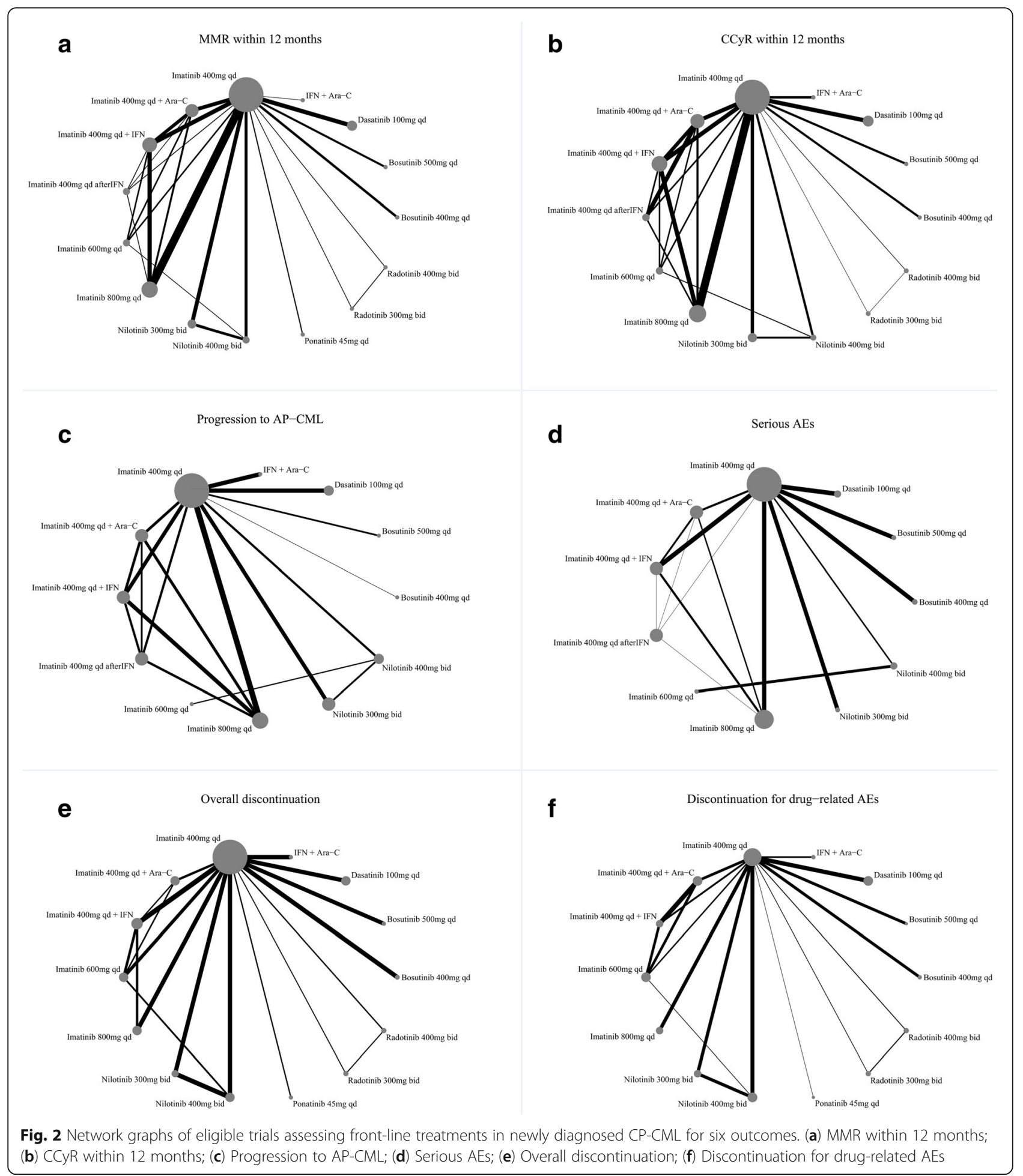

newer TKIs, such as dasatinib, radotinib, bosutinib, nilotinib and ponatinib, showed higher efficacy than imatinib in the first-line treatment of CP-CML patients, but the traditional treatment, such as IFN- $\alpha$ and Ara-C, suggested significantly lower efficacy when compared to TKIs. Low-dose nilotinib (300 mg BID) and radotinib
(300 mg BID) had higher efficacy than high-dose nilotinib (400 mg BID) and radotinib (400 mg BID), respectively. For overall discontinuation, traditional drugs showed higher dropout rate than TKIs. As for the discontinuation specially caused by drug-related adverse effects, most treatments showed lower acceptability when 
compared to standard-dose imatinib (400 mg QD), such as traditional drugs, newer TKIs and higher-dose imatinib (600 or $800 \mathrm{mg}$ QD). However, low-dose nilotinib (300 mg QD) generated higher tolerability than standard-dose imatinib (400 mg QD). Moreover, standarddose imatinib (400 mg QD) showed least probability of serious AEs when compared to other treatments. On the whole, statistical heterogeneity was moderate, although 95\% CIs were wide for several comparisons, which portrayed the small number of studies available for the pairwise comparison. Substantial heterogeneity was observed when comparing imatinib $400 \mathrm{mg}$ QD with nilotinib 400 mg BID $\left(\mathrm{I}^{2}=75.7 \%\right)$ for MMR or imatinib $400 \mathrm{mg}$ QD + Ara-C $\left(\mathrm{I}^{2}=87.3 \%\right)$ for CCyR. Nevertheless, there was no evidence showing heterogeneity in other pooled results of the direct comparisons for the six outcomes.

\section{Transitivity and consistency assessment}

As there were no observed significant clinical differences in distribution of effect modifiers between trials comparing different sets of interventions, we considered that the transitivity assumption was almost met (see Table 1 and Additional file 1: Table S1). All closed loops (networks of three comparisons that arise when collating studies involving different selections of competing treatments) were consistent, since the 95\% CIs of inconsistency factors (IF, the difference between the direct and indirect estimate for one of the comparisons in a particular loop) included zero. Furthermore, inconsistency test by the node-splitting method indicated that there was no significant inconsistency between direct and indirect evidence for nearly all $P$ values were higher than 0.05 (Additional file 1: Table S4). Analysis of inconsistency indicated that there was inconsistency in the loop for "CCyR" ("imatinib $400 \mathrm{mg}$ QD + Ara-C" - "imatinib $800 \mathrm{mg}$ QD"), another loop for "discontinuation for drug-related AEs" ("imatinib $400 \mathrm{mg}$ QD + Ara-C" "imatinib $600 \mathrm{mg}$ QD") and none for other four outcomes. Furtherly, we identified slight gender and sex difference across comparisons in these two loops, which may account for the inconsistency.

\section{Network estimation and cumulative ranking}

Pooled ORs with corresponding 95\% CIs for the efficacy and tolerability of different treatments from the network meta-analysis are shown in Table 2 and Additional file 1: Table S5. Rankograms that show the distribution of the probabilities of every treatment being ranked at each of the possible are presented in Additional file 2: Figure S2, and Table 3 presents all SUCRA values in terms of both efficacy and acceptability of each intervention. As for primary outcomes in MMR and CCyR, higher-dose imatinib (600 or $800 \mathrm{mg}$ QD) and newer TKIs, such as ponatinib, radotinib, bosutinib, nilotinib and dasatinib, were all highly effective in comparison to standard-dose imatinib, except that imatinib (600 mg QD) showed lower effective in CCyR. Obviously, the traditional treatment, such as IFN- $\alpha$ and Ara-C, generated significantly lower efficacy when compared to TKIs. Among newer

Table 2 Efficacy and tolerability of all treatments for CP-CML according to Bayesian network meta-analysis

\begin{tabular}{|c|c|c|c|c|c|c|c|c|c|c|c|c|c|c|}
\hline & $\begin{array}{c}1.05 \\
(0.65,1.67)\end{array}$ & $\begin{array}{l}1.96 \\
49,2.48)\end{array}$ & $\begin{array}{c}0.00 \\
.00,0.02)\end{array}$ & $\begin{array}{l}0.83 \\
.56,1.19)\end{array}$ & $\begin{array}{c}1.25 \\
.91,1.72)\end{array}$ & $\begin{array}{c}3.24 \\
.10,4.95)\end{array}$ & $\begin{array}{c}2.95 \\
(1.95,4.47)\end{array}$ & $\begin{array}{c}2.06 \\
.51,2.97)\end{array}$ & $\begin{array}{c}2.59 \\
.16,5.75)\end{array}$ & $\begin{array}{c}2.01 \\
(0.92,4.49)\end{array}$ & $\begin{array}{c}1.51 \\
(0.85,2.73)\end{array}$ & $\begin{array}{c}1.88 \\
1.03,3.47)\end{array}$ & $\begin{array}{c}6.67 \\
3.30,13.29)\end{array}$ & $\begin{array}{c}0.18 \\
(0.09,0.37)\end{array}$ \\
\hline & & & & & & & & & & & & & & \\
\hline $\begin{array}{c}2.32 \\
(1.00,5.25)\end{array}$ & $\begin{array}{l}2.23 \\
.57,8.59)\end{array}$ & $0 \mathrm{mg}$ qd & 0.00 & $(0.28,0.63)$ & $\begin{array}{c}0.64 \\
0.47,0.92)\end{array}$ & $\begin{array}{c}1.66 \\
(1.01,2.77)\end{array}$ & $\begin{array}{c}1.50 \\
(0.94,2.50)\end{array}$ & $\begin{array}{c}1.05 \\
(0.72,1.69)\end{array}$ & $\begin{array}{c}1.32 \\
(0.58,3.11)\end{array}$ & & & $\begin{array}{c}0.96 \\
(0.51,1.90)\end{array}$ & $\begin{array}{c}3.38 \\
(1.63,7.13)\end{array}$ & $\begin{array}{c}0.09 \\
(0.04,0.19)\end{array}$ \\
\hline $\begin{array}{c}3.25 \\
(0.85,12.64)\end{array}$ & $\begin{array}{c}3.13 \\
(0.56,16.64)\end{array}$ & $\begin{array}{c}1.41 \\
29,7.02)\end{array}$ & $N+$ Ara-C & $\begin{array}{c}249.74 \\
(40.67, \\
3429.57)\end{array}$ & $\begin{array}{c}380.31 \\
(62.20,5265.31)\end{array}$ & $\begin{array}{c}981.73 \\
(156.86 \\
13641.65)\end{array}$ & $\begin{array}{c}896.95 \\
(143.78 \\
12260.57)\end{array}$ & $\begin{array}{l}632.83 \\
(103.57 \\
8822.02)\end{array}$ & $\begin{array}{c}793.41 \\
(106.17 \\
12296.72)\end{array}$ & & $\begin{array}{l}466.09 \\
(70.27, \\
6540.84)\end{array}$ & $\begin{array}{l}578.18 \\
(87.08, \\
8398.08)\end{array}$ & & $\begin{array}{c}55.26 \\
(7.89,795.17\end{array}$ \\
\hline $\begin{array}{c}4.94 \\
(1.68,12.42)\end{array}$ & $\begin{array}{c}4.82 \\
(1.27,13.22)\end{array}$ & $\begin{array}{l}2.14 \\
.55,7.18)\end{array}$ & $\begin{array}{c}1.53 \\
(0.26,7.09)\end{array}$ & $\begin{array}{c}\text { Imatinib } \\
400 \mathrm{mg} \mathrm{qd}+ \\
\text { Ara-C }\end{array}$ & $\begin{array}{c}1.51 \\
(1.03,2.30)\end{array}$ & $\begin{array}{c}3.90 \\
(2.25,6.94)\end{array}$ & $\begin{array}{c}3.56 \\
(2.09,6.24)\end{array}$ & $\begin{array}{c}2.51 \\
(1.54,4.32)\end{array}$ & $\begin{array}{c}3.10 \\
(1.31,7.75)\end{array}$ & $\begin{array}{c}2.43 \\
(1.02,5.94)\end{array}$ & $\begin{array}{c}1.83 \\
(0.93,3.72)\end{array}$ & $\begin{array}{c}2.28 \\
(1.16,4.72)\end{array}$ & $\begin{array}{c}7.98 \\
(3.67,17.70)\end{array}$ & $\begin{array}{c}0.22 \\
(0.10,0.47)\end{array}$ \\
\hline $\begin{array}{c}14.05 \\
(4.15,45.81)\end{array}$ & $\begin{array}{c}13.59 \\
(3.64,45.20)\end{array}$ & $\begin{array}{c}6.09 \\
(1.37,25.05)\end{array}$ & \begin{tabular}{c|}
4.34 \\
$(0.70,25.58)$ \\
\end{tabular} & \begin{tabular}{c|}
2.80 \\
$(0.96,10.34)$ \\
\end{tabular} & $\begin{array}{c}\text { Imatinib } 400 \mathrm{~m} \\
\mathrm{qd}+\mathrm{IFN}\end{array}$ & $\begin{array}{c}2.58 \\
(1.51,4.35)\end{array}$ & $\begin{array}{c}2.34 \\
(1.41,3.87)\end{array}$ & $\begin{array}{c}1.65 \\
(1.06,2.65)\end{array}$ & $\begin{array}{c}2.03 \\
(0.87,4.91)\end{array}$ & $\begin{array}{c}1.60 \\
(0.69,3.77)\end{array}$ & $\begin{array}{c}1.21 \\
(0.62,2.36)\end{array}$ & $\begin{array}{c}1.50 \\
(0.77,2.99)\end{array}$ & $\begin{array}{c}5.23 \\
(2.46,11.34)\end{array}$ & $\begin{array}{c}0.14 \\
(0.07,0.29)\end{array}$ \\
\hline $\begin{array}{c}1.28 \\
(0.49,3.88)\end{array}$ & $\begin{array}{c}1.23 \\
(0.31,5.34) \\
\end{array}$ & $\begin{array}{c}0.55 \\
.16,2.27) \\
\end{array}$ & $\begin{array}{c}0.39 \\
(0.08,2.36) \\
\end{array}$ & $\begin{array}{c}0.26 \\
(0.07,1.27) \\
\end{array}$ & $\begin{array}{c}0.09 \\
(0.02,0.50)\end{array}$ & & $\begin{array}{c}0.91 \\
(0.57,1.50) \\
\end{array}$ & $\begin{array}{c}0.64 \\
(0.38,1.14)\end{array}$ & $\begin{array}{c}0.79 \\
(0.33,1.99)\end{array}$ & $\begin{array}{c}0.62 \\
(0.26,1.55) \\
\end{array}$ & $\begin{array}{c}0.47 \\
(0.23,0.98)\end{array}$ & $\begin{array}{c}0.58 \\
(0.28,1.25) \\
\end{array}$ & $\begin{array}{c}2.03 \\
(0.91,4.69)\end{array}$ & $\begin{array}{c}0.06 \\
(0.02,0.13)\end{array}$ \\
\hline $\begin{array}{c}2.01 \\
(0.94,5.50)\end{array}$ & $\begin{array}{c}1.94 \\
.65,6.77)\end{array}$ & $\begin{array}{c}0.86 \\
.29,3.17) \\
\end{array}$ & $\begin{array}{c}0.62 \\
(0.14,3.32) \\
\end{array}$ & $\begin{array}{l}0 \\
1.75)\end{array}$ & $\begin{array}{c}0.14 \\
(0.04,0.66) \\
\end{array}$ & $\begin{array}{c}1.56 \\
(0.54,4.89) \\
\end{array}$ & & \begin{tabular}{|c|}
0.70 \\
$(0.42,1.21)$ \\
\end{tabular} & $\begin{array}{c}0.89 \\
(0.36,2.15)\end{array}$ & $\begin{array}{c}0.68 \\
(0.28,1.68) \\
\end{array}$ & $\begin{array}{c}0.51 \\
(0.25,1.06) \\
\end{array}$ & $\begin{array}{c}0.64 \\
(0.31,1.33) \\
\end{array}$ & $\begin{array}{c}2.26 \\
(1.01,4.98) \\
\end{array}$ & $\begin{array}{c}0.06 \\
(0.03,0.14) \\
\end{array}$ \\
\hline $\begin{array}{c}1.42 \\
(0.71,2.81)\end{array}$ & $\begin{array}{c}1.36 \\
(0.35,4.64)\end{array}$ & $\begin{array}{c}0.61 \\
(0.21,1.77)\end{array}$ & $\begin{array}{c}0.43 \\
(0.09,1.95)\end{array}$ & $\begin{array}{c}0.29 \\
(0.09,1.07)\end{array}$ & $\begin{array}{c}0.10 \\
(0.02,0.41)\end{array}$ & $\begin{array}{c}1.11 \\
(0.29,3.55)\end{array}$ & $\begin{array}{c}0.71 \\
(0.20,1.90)\end{array}$ & $\begin{array}{l}\text { Dasatinib } \\
100 \mathrm{mg} \mathrm{qd}\end{array}$ & $\begin{array}{c}1.25 \\
(0.52,2.96)\end{array}$ & $\begin{array}{c}0.97 \\
(0.40,2.25)\end{array}$ & $\begin{array}{c}0.74 \\
(0.36,1.42)\end{array}$ & $\begin{array}{c}0.91 \\
(0.45,1.78)\end{array}$ & $\begin{array}{c}3.22 \\
(1.45,6.82)\end{array}$ & $\begin{array}{c}0.09 \\
(0.04,0.19)\end{array}$ \\
\hline $\begin{array}{c}1.54 \\
(0.30,9.48) \\
\end{array}$ & $\begin{array}{c}1.49 \\
(0.20,11.59)\end{array}$ & \begin{tabular}{c|}
0.66 \\
$(0.11,4.68)$ \\
\end{tabular} & $\begin{array}{c}0.47 \\
(0.06,4.88) \\
\end{array}$ & $\begin{array}{c}0.31 \\
(0.05,2.61) \\
\end{array}$ & $\begin{array}{c}0.11 \\
(0.01,0.97) \\
\end{array}$ & $\begin{array}{c}1.21 \\
(0.16,9.21) \\
\end{array}$ & $\begin{array}{c}0.78 \\
(0.11,5.33) \\
\end{array}$ & \begin{tabular}{c|}
1.09 \\
$(0.19,7.68)$ \\
\end{tabular} & $\begin{array}{l}\text { Radotinib } \\
300 \mathrm{mg} \text { bid }\end{array}$ & $\begin{array}{c}0.78 \\
(0.35,1.68)\end{array}$ & $\begin{array}{c}0.58 \\
(0.22,1.59) \\
\end{array}$ & $\begin{array}{c}0.72 \\
(0.27,1.98)\end{array}$ & $\begin{array}{c}2.58(0.87, \\
7.30)\end{array}$ & $\begin{array}{c}0.07(0.02, \\
0.21)\end{array}$ \\
\hline $\begin{array}{c}4.14 \\
(0.92,21.57) \\
\end{array}$ & $\begin{array}{c}3.98 \\
(0.59,28.95) \\
\end{array}$ & $\begin{array}{c}1.79 \\
(0.32,11.04)\end{array}$ & \begin{tabular}{c|}
1.27 \\
$(0.16,10.94)$ \\
\end{tabular} & $\begin{array}{c}0.85 \\
(0.15,6.28)\end{array}$ & $\begin{array}{c}0.30 \\
(0.04,2.28)\end{array}$ & $\begin{array}{c}3.24 \\
(0.49,21.49)\end{array}$ & $\begin{array}{c}2.07 \\
(0.33,12.57)\end{array}$ & $\begin{array}{c}2.97 \\
(0.56,17.20)\end{array}$ & $\begin{array}{c}2.65 \\
(0.61,12.05) \\
\end{array}$ & $\begin{array}{l}\text { Radotinib } \\
400 \mathrm{mg} \text { bid }\end{array}$ & $\begin{array}{c}0.75 \\
(0.28,2.03) \\
\end{array}$ & $\begin{array}{c}0.94 \\
(0.35,2.55)\end{array}$ & $\begin{array}{c}3.27 \\
(1.13,9.55)\end{array}$ & $\begin{array}{c}0.09 \\
(0.03,0.27) \\
\end{array}$ \\
\hline $\begin{array}{c}1.53 \\
(0.43,5.60)\end{array}$ & $\begin{array}{c}1.47 \\
(0.28,7.50)\end{array}$ & $\begin{array}{c}0.66 \\
(0.15,2.97)\end{array}$ & $\begin{array}{c}0.47 \\
(0.07,3.10)\end{array}$ & $\begin{array}{c}0.31 \\
(0.07,1.71)\end{array}$ & $\begin{array}{c}0.11 \\
(0.02,0.65)\end{array}$ & $\begin{array}{c}1.19 \\
(0.22,5.52)\end{array}$ & $\begin{array}{c}0.77 \\
(0.14,3.23)\end{array}$ & \begin{tabular}{c|}
1.08 \\
$(0.25,4.60)$ \\
\end{tabular} & $\begin{array}{c}0.99 \\
(0.12,8.04) \\
\end{array}$ & $\begin{array}{c}0.37 \\
(0.05,2.62)\end{array}$ & $\begin{array}{l}\text { Bosutinib } \\
400 \mathrm{mg} \mathrm{qd}\end{array}$ & $\begin{array}{c}1.24 \\
(0.54,2.89)\end{array}$ & $\begin{array}{c}4.40 \\
(1.75,10.79)\end{array}$ & $\begin{array}{c}0.12 \\
(0.05,0.30)\end{array}$ \\
\hline $\begin{array}{c}4.18 \\
(1.23,16.04)\end{array}$ & $\begin{array}{c}4.10 \\
(0.71,21.04)\end{array}$ & $\begin{array}{c}1.82 \\
(0.39,9.21) \\
\end{array}$ & $\begin{array}{c}1.32 \\
(0.20,8.18) \\
\end{array}$ & $\begin{array}{c}0.85 \\
(0.18,4.79) \\
\end{array}$ & $\begin{array}{c}0.30 \\
(0.05,1.75)\end{array}$ & $\begin{array}{c}3.34 \\
(0.60,15.91)\end{array}$ & $\begin{array}{c}2.12 \\
(0.41,8.98)\end{array}$ & $\begin{array}{c}2.97 \\
(0.71,13.61) \\
\end{array}$ & \begin{tabular}{c|}
2.70 \\
$(0.33,21.78)$ \\
\end{tabular} & \begin{tabular}{c|}
1.01 \\
$(0.14,7.37)$ \\
\end{tabular} & $\begin{array}{c}2.76 \\
(0.45,17.06) \\
\end{array}$ & $\begin{array}{l}\text { Bosutinib } \\
500 \mathrm{mg} \mathrm{qd}\end{array}$ & $\begin{array}{c}3.54 \\
(1.40,8.63)\end{array}$ & $\begin{array}{c}0.10 \\
(0.04,0.25)\end{array}$ \\
\hline $\begin{array}{c}8.86 \\
(1.48,87.98) \\
\end{array}$ & $\begin{array}{c}8.46 \\
(0.98,104.39)\end{array}$ & $\begin{array}{c}3.82 \\
(0.54,45.38) \\
\end{array}$ & \begin{tabular}{c|}
2.77 \\
$(0.28,38.49)$ \\
\end{tabular} & $\begin{array}{c}1.81 \\
(0.24,23.29) \\
\end{array}$ & $\begin{array}{c}0.63 \\
(0.07,8.06)\end{array}$ & $\begin{array}{c}6.80 \\
(0.82,87.32)\end{array}$ & $\begin{array}{c}4.32 \\
(0.54,48.99)\end{array}$ & $\begin{array}{c}6.29 \\
(0.93,73.09) \\
\end{array}$ & \begin{tabular}{c|}
5.75 \\
$(0.47,95.73)$ \\
\end{tabular} & \begin{tabular}{c|}
2.14 \\
$(0.19,32.08)$ \\
\end{tabular} & $\begin{array}{c}5.64 \\
(0.64,78.19) \\
\end{array}$ & $\begin{array}{c}2.05 \\
(0.24,30.14) \\
\end{array}$ & $\begin{array}{l}\text { Ponatinib } \\
45 \mathrm{mg} \mathrm{qd} \\
\end{array}$ & $\begin{array}{c}0.03 \\
(0.01,0.08)\end{array}$ \\
\hline & - & - & - & - & - & - & . & - & & - & - & - & - & \\
\hline
\end{tabular}


Table 3 Surface under the cumulative ranking curve (SUCRA) data for six outcomes

\begin{tabular}{|c|c|c|c|c|c|c|}
\hline \multirow[t]{2}{*}{ Treatment } & \multicolumn{6}{|c|}{ Surface Under the Cumulative Ranking Curve (SUCRA) } \\
\hline & MMR within 12 months & CCyR within 12 months & $\begin{array}{l}\text { Progression } \\
\text { to AP-CML }\end{array}$ & $\begin{array}{l}\text { Overall } \\
\text { Discontinuation }\end{array}$ & $\begin{array}{l}\text { Discontinuation } \\
\text { for Drug-related AEs }\end{array}$ & Serious AEs \\
\hline Bosutinib $400 \mathrm{mg}$ qd & 0.428 & 0.684 & 0.259 & 0.318 & 0.346 & 0.572 \\
\hline Bosutinib $500 \mathrm{mg}$ qd & 0.570 & 0.416 & 0.872 & 0.520 & 0.719 & 0.581 \\
\hline Dasatinib $100 \mathrm{mg}$ qd & 0.624 & 0.736 & 0.302 & 0.337 & 0.312 & 0.632 \\
\hline IFN + Ara-C & 0.000 & 0.000 & 0.719 & 0.726 & 0.476 & - \\
\hline Imatinib 400 mg qd & 0.209 & 0.335 & 0.406 & 0.383 & 0.158 & 0.481 \\
\hline Imatinib 400 mg qd + Ara-C & 0.172 & 0.340 & 0.482 & 0.903 & 0.769 & 0.483 \\
\hline Imatinib 400 mg qd + IFN & 0.321 & 0.411 & 0.350 & 0.653 & 0.958 & 0.488 \\
\hline Imatinib 400 mg qd after IFN & 0.071 & 0.076 & - & - & - & 0.159 \\
\hline Imatinib 600 mg qd & 0.449 & 0.311 & 0.254 & 0.352 & 0.187 & 0.477 \\
\hline Imatinib 800 mg qd & 0.682 & 0.730 & 0.388 & 0.525 & 0.576 & 0.528 \\
\hline Nilotinib 300 mg bid & 0.791 & 0.759 & 0.301 & 0.372 & 0.234 & 0.543 \\
\hline Nilotinib 400 mg bid & 0.839 & 0.781 & 0.293 & 0.416 & 0.440 & 0.556 \\
\hline Ponatinib 45 mg qd & 0.997 & - & 0.419 & 0.649 & 0.837 & - \\
\hline Radotinib $300 \mathrm{mg}$ bid & 0.750 & 0.895 & - & 0.283 & 0.343 & - \\
\hline Radotinib 400 mg bid & 0.596 & 0.538 & - & 0.561 & 0.688 & - \\
\hline
\end{tabular}

("-" means "can't be evaluated")

TKIs, ponatinib was identified to be the most effective, and nilotinib, radotinib, dasatinib as well as bosutinib showed relatively higher efficacy. Nilotinib (300 or 400 mg BID), dasatinib (100 mg QD), low-dose bosutinib (400 mg QD) and higher-dose imatinib (600 or $800 \mathrm{mg}$ QD) showed lower probability of disease progression to AP-CML. As for serious AEs, there were no significant difference among studied treatments, SUCRAs of which ranged from 0.477 to 0.632 , except that SUCRA for imatinib $400 \mathrm{mg}$ QD after IFN was 0.159 . In terms of discontinuation for drug-related AEs, standard-dose imatinib (400 mg QD) was the most tolerable treatment, and nilotinib (300 or $400 \mathrm{mg}$ BID), dasatinib (100 mg QD), higher-dose imatinib (600 or $800 \mathrm{mg}$ QD), and low-dose radotinib (300 mg BID) were better than other treatments. Traditional drugs and newer TKIs showed lower acceptability than imatinib, and the drug toxicity were positively associated with drug dose. But as for overall discontinuation, low-dose radotinib (300 mg BID) suggested lowest treatment discontinuation, and imatinib (400 or $600 \mathrm{mg}$ QD), nilotinib (300 or $400 \mathrm{mg}$ BID), and low-dose bosutinib (400 mg QD) showed relatively lower dropout rate than other treatments.

\section{Cluster analysis}

Utilizing the SUCRA values, we displayed a clustered ranking plot of these treatments in the two dimensions of the $\mathrm{x}$-axis (efficacy as higher MMR within 12 months) and the $y$-axis (tolerability as less discontinuation for drug-related AEs) in Fig. 3. Cluster analysis demonstrated the division of treatments into eight distinct groups. One cluster of interventions, which includes nilotinib (300 or 400 $\mathrm{mg}$ BID), radotinib (300 mg BID) and dasatinib (100 mg QD), has relatively higher efficacy and tolerability compared with other treatments. Ponatinib (45 mg QD) and imatinib (400 mg QD) suggested highest efficacy and tolerability, respectively.

\section{Reporting bias}

The funnel plots seemed to be approximately symmetrical for four outcomes (MMR, CCyR, progression to $\mathrm{AP}-\mathrm{CML}$ and overall discontinuation), but rather asymmetrical for serious AEs and discontinuation for drugrelated AEs, which suggests that several studied treatments were favored more in small trials (Additional file 2: Figure S3).

\section{Discussion}

To our knowledge, this was the first to comprehensively assess the comparative efficacy and tolerability of almost all front-line treatments for newly diagnosed CP-CML patients, involving 21 RCTs (10,187 patients). Our study suggests both statistically and clinically significant differences among front-line treatments of newly diagnosed CP-CML patients.

Regarding the efficacy, we focused on three important indicators, early major molecular response (MMR, $\leq 0.1 \%$ BCR-ABL1[IS]), complete cytogenetic response (CCyR, $\leq 1 \%$ BCR-ABL1[IS]) and disease progression to APCML. The prognostic significance of early MMR and 


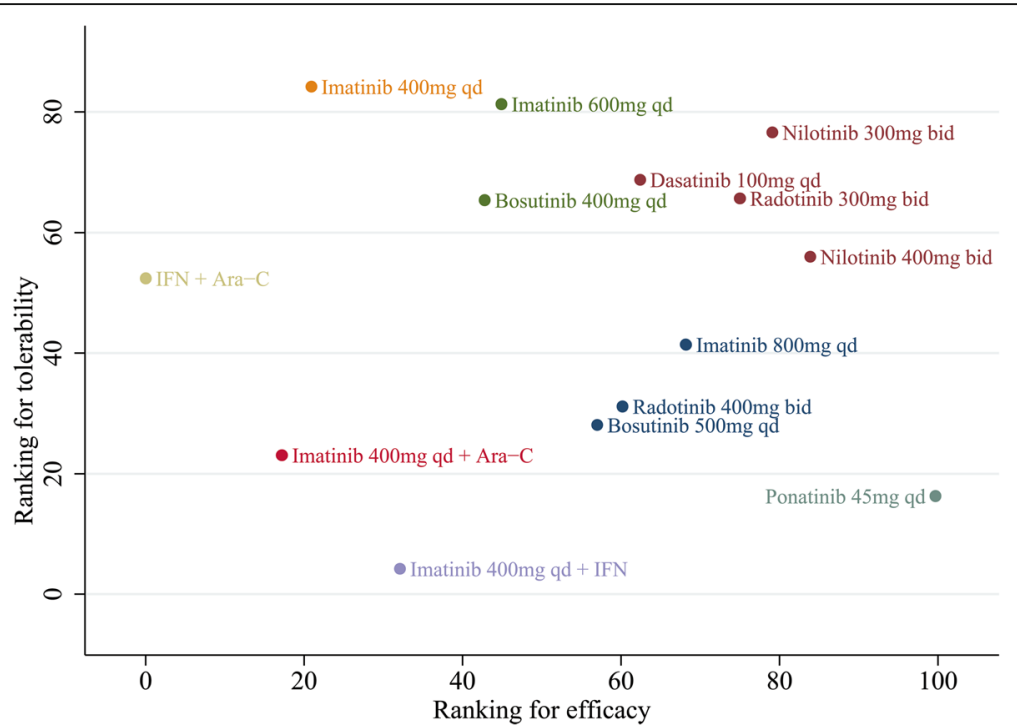

Fig. 3 Comprehensive ranking (efficacy and tolerability) of front-line treatments in newly diagnosed CP-CML. (Efficacy is evaluated as MMR within 12 months and tolerability is evaluated as less discontinuation for drug-related AEs)

CCyR after first-line treatment has been evaluated in several studies [55-57]. Achievement of MMR and CCyR within 12 months is an established prognostic indicator of long-term survival. Furthermore, achievement of MMR within 12 months is associated with a very low probability of subsequent disease progression and a high likelihood of achieving a subsequent deep molecular response. In addition, disease progression to AP-CML while on drug therapy usually has worse prognosis than de novo AP-CML. In terms of tolerability, we focused on serious AEs, overall discontinuation and discontinuation due to drug-related AEs during therapy at early stage. Serious AEs refer to adverse effects in higher (3 or 4) grade, including non-hematological and hematological adverse effects. Overall treatment discontinuation is influenced by many factors, including drug-related AEs, refusal, failure to achieve complete hematologic response, relapse and disease progression. However, as for discontinuation for drug-related AEs, it specifically refers to the safety of the therapeutic drug, which is more likely to reveal actual drug tolerability.

The treatment of CML has undergone an evolution with the advent of imatinib, which has significantly changed the natural history of the disease with an increase of 10 -year OS from 10 to $20 \%$ to $80-90 \%$ [6]. According to our study, standard-dose imatinib (400 mg QD) proves to be of greater efficacy than traditional drugs or the combination therapy of imatinib with traditional drugs. However, several patients may have resistance and/or intolerance to imatinib and these patients require further treatment options, such as second-generation TKIs and ponatinib.
Our analysis suggests that patients receiving nilotinib as initial treatment achieve faster cytogenetic and molecular responses with a lower rate of transformation to more advanced phases of CML and relatively higher drug tolerability. Therefore, nilotinib might be an excellent choice as front-line therapy in CP-CML due to greater potency and selectivity for BCR-ABL1 kinase inhibition and higher tolerability. However, some observed long-term toxicity effects (particularly cardiovascular events and diabetes mellitus) suggest that nilotinib should be used with caution in patients with cardiovascular risk factors and metabolic syndrome [58]. Additionally, dasatinib (100 mg QD) and radotinib (300 mg BID) demonstrates almost similar efficacy and acceptability as nilotinib. Nilotinib, dasatinib and bosutinib are second-generation TKIs approved in many countries for CML following many international multicenter trials, but radotinib is currently approved only in Korea for this indication. High-dose imatinib (800 mg QD), radotinib (400 mg BID) and bosutinib (500 mg QD) demonstrates very low tolerability, thus they are recommended only for patients in accelerated phase/blast phase or with suboptimal CML-CP response. Management of adverse events should be carried out to avoid compromising the clinical efficacy. Ponatinib, the most recently approved TKI, was found to be of greatest probability of MMR within 12 months, but relatively higher tendency of treatment dropout. Ponatinib has demonstrated efficacy in patients with refractory CML, but is associated with an increased risk of arterial hypertension, sometimes severe, and serious arterial occlusive and venous thromboembolic events [16]. CML patients, with presence of the T315I mutation, resistance or intolerance to other TKIs, may be 
an appropriate candidate for ponatinib therapy [16, 59]. As mentioned before, TKI drugs that can achieve faster MMR are usually associated with low disease progression and high likelihood of achieving a deep molecular response. Our study suggests that newer-generation TKIs generate faster molecular and cytogenic response. The primary goal of TKI therapy for CP-CML patients is prevention of disease progression, which is more common in patients with intermediate- or high-risk score. Therefore, newer-generation TKI drugs with a low probability of disease progression are preferred for patients with intermediate or high risk. Almost all TKIs are more tolerated than traditional drugs, and the difference in their potential toxicity profiles may influence the selection of initial therapy. In terms of starting does of TKI drugs, only patients who can actually tolerate the potential drug toxicity are recommend to receive high-dose therapy.

The NCCN guideline recommends imatinib (400 mg QD) and second-generation TKIs (dasatinib (100 mg QD), nilotinib (300 mg BID) and bosutinib (400 mg QD) as appropriate options for front-line TKI therapy for patients with CP-CML across all risk scores [15]. Additionally, the previous two meta-analysis concluded that nilotinib seems to be the best choice for first-line therapy in CML patients, compared with the currently available TKIs on the international market [11, 12]. Unlike the previous study, our study introduced the analysis of new first-line TKI therapy, such as radotinib and ponatinib. One cluster of treatments including nilotinib (300 or $400 \mathrm{mg}$ BID), radotinib (300 mg BID) and dasatinib (100 mg QD), demonstrates relatively greater efficacy and tolerability than other treatments. Although ponatinb suggests highest efficacy in early cytogenetic and molecular responses, its great side-effects and weak tolerability can't be ignored. High-dose imatinib $(800 \mathrm{mg}$ QD), radotinib (400 $\mathrm{mg}$ BID) and bosutinib (500 mg QD) are recommended only for patients in accelerated phase/blast phase or with suboptimal CML-CP response. These results may have potential clinical implications, which provide useful information for clinical decisionmaking and should be considered in the development of clinical practice guidelines. But during the clinical practice, the selection of front-line TKI therapy should be based on several factors, such as risk score, patient's stage, ability to tolerate therapy, drug toxicity and the present comorbid conditions [15].

Strictly speaking, we designed this NMA as standardized by the PRISMA principle and conducted it carefully to minimize errors and ensure the validity of findings from all eligible trials. Nevertheless, there are also several limitations to our research due to its nature or design. Firstly, we just included limited number of trials, and several drugs (such as radotinib, ponatinib) were only used in limited countries and areas. Secondly, although MMR and
CCyR are important prognostic indicator, which are able to represent the disease prognosis to some degree, we were unable to perform the NMA of some important prognostic outcomes directly for insufficient follow-up data, such as overall survival (OS) and progression-free survival (PFS). Thirdly, we didn't take the cost-effective element into consideration, thus further cost-effectiveness analyses are necessary to evaluate the economic feasibility. Fourthly, some estimated results of this NMA relied on indirect comparisons despite of no evidence suggesting obvious inconsistency. Therefore, the application of our study should take into account any limitations of the analysis and the specific clinical situation.

\section{Conclusions}

In conclusion, one cluster of treatments including nilotinib (300 or $400 \mathrm{mg}$ BID), radotinib (300 mg BID) and dasatinib (100 mg QD), might be an excellent choice as front-line therapy in CP-CML due to superior efficacy and tolerability than other treatments. High-dose TKI therapies are recommended only for patients in accelerated phase/blastic phase or with suboptimal CML-CP response, and management of adverse events should be carried out to avoid compromising the clinical efficacy. Results from our study have potential clinical implications, which provide useful information for clinical decision-making and should be considered in the development of clinical practice guidelines. In the future, more clinical trials will be necessary to investigate further role of TKI therapy in CPCML patients. Furthermore, new TKI treatments with higher efficacy or acceptability than the existing treatments are urgently needed to be explored.

\section{Additional files}

Additional file 1: Table S1. Supplementary characteristics of trials and patients in the 21 eligible RCTs (10,187 patients). Table S2. Risk of bias assessment. Table S3. Efficacy and tolerability of all treatments according to pairwise estimates. Table S4. Inconsistency evaluation. Table S5. Efficacy and tolerability of all treatments according to Bayesian network meta-analysis. Appendix 1 WinBUGS codes (PDF 263 kb)

Additional file 2: Figure S1. Risk of bias assessment (a) Risk of bias graph; (b) Risk of bias summary. Figure S2. Rankograms for six outcomes (a) MMR within 12 months; (b) CCyR within 12 months; (c) Progression to AP-CML; (d) Serious AEs; (e) Overall discontinuation; (f) Discontinuation for drug-related AEs. Figure S3. Funnel plots for six outcomes (a) MMR within 12 months; (b) CCyR within 12 months; (c) Progression to AP-CML; (d) Serious AEs; (e) Overall discontinuation; ( $f$ ) Discontinuation for drugrelated AEs (PDF $2981 \mathrm{~kb}$ )

\section{Abbreviations}

ABL1: Abelson murine leukemia 1; AE: Adverse events; Allo-SCT: Allogeneic stem cell transplant; AP-CML: Accelerated-phase chronic myeloid leukemia; Ara-C: Cytosine arabinoside; ATP: Adenosine triphosphate; BCR: Breakpoint cluster region; CCyR: Complete cytogenetic response; Cl: Confidence interval; CP-CML: Chronic-phase chronic myeloid leukemia; ECOG: Eastern

Cooperative Oncology Group; FISH: Fluorescence in situ hybridization; Hu: Hydroxyurea; IF: Inconsistency factor; IFN-a: Interferon-alfa; MMR: Major molecular response; MPN: Myeloproliferative neoplasm; NCl: National Cancer 
Institute; NMA: Network meta-analysis; OR: Odds ratio; OS: Overall survival; PFS: Progression-free survival; Ph: Philadelphia chromosome; PRISMA: Preferred Reporting Items for Systematic Reviews and MetaAnalyses; RCT: Randomized controlled trial; RT-qPCR: Reverse transcriptionquantitative polymerase chain reaction; SUCRA: Surface under the cumulative ranking; TKl: Tyrosine kinase inhibitors

\section{Acknowledgments}

We would like to thank all researchers for their contributions.

\section{Authors' contributions}

$\mathrm{TL}, \mathrm{ZH}, \mathrm{PYZ}, \mathrm{LCG}, \mathrm{JHW}, \mathrm{XM}, \mathrm{MH}$ and $\mathrm{HY}$ conceived and designed this study. $\mathrm{TL}, \mathrm{XM}, \mathrm{ZH}, \mathrm{PYZ}$ and $\mathrm{MH}$ collected and analyzed the data. $\mathrm{TL}$ and $\mathrm{MH}$ wrote the paper. All authors reviewed the paper, and approved the final manuscript

\section{Funding}

This work was supported by grants from the National Natural Science Foundation of China (No. 81770132 for Yu Hu and No. 81570116 for Heng Mei) and the Science and Technology Department of Hubei Province (No. 2018ACA141 for Yu Hu). The content is solely the responsibility of the authors and does not necessarily represent the National Natural Science Foundation of China and the Science and Technology Department of Hubei Province. The funding agency did not have a role in the design of the study, the collection, analysis, and interpretation of data, or the writing of the manuscript.

\section{Availability of data and materials}

The authors declare that all data supporting the findings of this study are available within the article and the enrolled articles for meta-analysis.

\section{Ethics approval and consent to participate}

Not Applicable. Because our study is a network meta-analysis, the ethics approval and consent to participate is not relevant to our article type.

\section{Consent for publication}

Not Applicable. There are no details on individuals reported within the manuscript, so we don't have the consent for publication.

\section{Competing interests}

The authors declare that they have no competing interests.

\section{Author details}

${ }^{1}$ Institute of Hematology, Union Hospital, Tongji Medical College, Huazhong University of Science and Technology, 1277 Jiefang Road, Wuhan 430022, Hubei, China. ${ }^{2}$ Hubei clinical medical center of cell therapy for neoplastic disease, Wuhan, Hubei, China. ${ }^{3}$ Collaborative Innovation Center of Hematology, Huazhong University of Science and Technology, Wuhan, Hubei, China. ${ }^{4}$ Instisute of Pancreatic Surgery, Union Hospital, Tongji Medical College, Huazhong University of Science and Technology, 1227 Jiefang road, Wuhan 430022, Hubei, China. ${ }^{5}$ Instisute of Orthopedics, Union Hospital, Tongji Medical College, Huazhong University of Science and Technology, 1227 Jiefang road, Wuhan 430022 „, Hubei, China.

\section{Received: 30 January 2019 Accepted: 14 August 2019} Published online: 28 August 2019

\section{References}

1. Apperley JF. Chronic myeloid leukaemia. Lancet. 2015;385(9976):1447-59.

2. Jabbour E, Kantarjian H. Chronic myeloid leukemia: 2018 update on diagnosis, therapy and monitoring. Am J Hematol. 2018;93(3):442-59.

3. Pasic I, Lipton JH. Current approach to the treatment of chronic myeloid leukaemia. Leuk Res. 2017;55:65-78.

4. Pallera A, Altman JK, Berman E, Abboud CN, Bhatnagar B, Curtin P, DeAngelo DJ, Gotlib J, Hagelstrom RT, Hobbs G, et al. NCCN guidelines insights: chronic myeloid leukemia, version 1.2017. J Natl Compr Cancer Netw. 2016;14(12):1505-12.

5. Silver RT, Woolf SH, Hehlmann R, Appelbaum FR, Anderson J, Bennett C, Goldman JM, Guilhot F, Kantarjian HM, Lichtin AE, et al. An evidence-based analysis of the effect of busulfan, hydroxyurea, interferon, and allogeneic bone marrow transplantation in treating the chronic phase of chronic myeloid leukemia: developed for the American Society of Hematology. Blood. 1999;94(5):1517-36.

6. Huang $X$, Cortes J, Kantarjian H. Estimations of the increasing prevalence and plateau prevalence of chronic myeloid leukemia in the era of tyrosine kinase inhibitor therapy. Cancer. 2012;118(12):3123-7.

7. Gurion R, Raanani P, Vidal L, Leader A, Gafter-Gvili A. First line treatment with newer tyrosine kinase inhibitors in chronic myeloid leukemia associated with deep and durable molecular response - systematic review and meta-analysis. Acta Oncol (Stockholm, Sweden). 2016;55(9-10):1077-83.

8. Signorovitch J, Ayyagari R, Reichmann WM, Wu EQ, Chen L. Major molecular response during the first year of dasatinib, imatinib or nilotinib treatment for newly diagnosed chronic myeloid leukemia: a network meta-analysis. Cancer Treat Rev. 2014;40(2):285-92.

9. Hoffmann VS, Hasford J, Deininger M, Cortes J, Baccarani M, Hehlmann R. Systematic review and meta-analysis of standard-dose imatinib vs. highdose imatinib and second generation tyrosine kinase inhibitors for chronic myeloid leukemia. J Cancer Res Clin Oncol. 2017;143(7):1311-8.

10. Yun S, Vincelette ND, Segar JM, Dong Y, Shen Y, Kim DW, Abraham I. Comparative effectiveness of newer tyrosine kinase inhibitors versus Imatinib in the first-line treatment of chronic-phase chronic myeloid leukemia across risk groups: a systematic review and meta-analysis of eight randomized trials. Clin Lymphoma Myeloma Leuk. 2016;16(6):e85-94.

11. Chen KK, Du TF, Wu KS, Yang W. First-line treatment strategies for newly diagnosed chronic myeloid leukemia: a network meta-analysis. Cancer Manag Res. 2018;10:3891-910.

12. Fachi MM, Tonin FS, Leonart LP, Aguiar KS, Lenzi L, Figueiredo BC, Fernandez-Llimos F, Pontarolo R. Comparative efficacy and safety of tyrosine kinase inhibitors for chronic myeloid leukaemia: A systematic review and network meta-analysis. Eur J Cancer (Oxford, England : 1990). 2018;104:9-20.

13. Chaimani A, Caldwell DM, Li T, Higgins JPT, Salanti G. Additional considerations are required when preparing a protocol for a systematic review with multiple interventions. J Clin Epidemiol. 2017:83:65-74.

14. Hutton B, Salanti G, Caldwell DM, Chaimani A, Schmid CH, Cameron C, loannidis JP, Straus S, Thorlund K, Jansen JP, et al. The PRISMA extension statement for reporting of systematic reviews incorporating network metaanalyses of health care interventions: checklist and explanations. Ann Intern Med. 2015;162(11):777-84.

15. Network: NCCN clinical practice guidelines in oncology: chronic myelogenous leukemia version 2. 2018:2017.

16. Muller MC, Cervantes F, Hjorth-Hansen H, Janssen J, Milojkovic D, Rea D, Rosti G. Ponatinib in chronic myeloid leukemia (CML): consensus on patient treatment and management from a European expert panel. Crit Rev Oncol Hematol. 2017;120:52-9.

17. Higgins JP, Green S. Cochrane Handbook for Systematic Reviews of Interventions Version 5.1.0. Naunyn Schmiedebergs Arch Exp Pathol Pharmakol. 2011;5(2):S38

18. Li T, Puhan MA, Vedula SS, Singh S, Dickersin K. Network meta-analysishighly attractive but more methodological research is needed. BMC Med. 2011;9:79.

19. Jansen JP, Naci H. Is network meta-analysis as valid as standard pairwise meta-analysis? It all depends on the distribution of effect modifiers. BMC Med. 2013;11:159.

20. Dias S, Welton NJ, Caldwell DM, Ades AE. Checking consistency in mixed treatment comparison meta-analysis. Stat Med. 2010;29(7-8):932-44.

21. Hochhaus A, Larson RA, Guilhot F, Radich JP, Branford S, Hughes TP, Baccarani M, Deininger MW, Cervantes F, Fujihara S, et al. Long-term outcomes of Imatinib treatment for chronic myeloid leukemia. N Engl J Med. 2017;376(10):917-27.

22. Hughes TP, Kaeda J, Branford S, Rudzki Z, Hochhaus A, Hensley ML, Gathmann I, Bolton AE, van Hoomissen IC, Goldman JM, et al. Frequency of major molecular responses to imatinib or interferon alfa plus cytarabine in newly diagnosed chronic myeloid leukemia. N Engl J Med. 2003;349(15): 1423-32.

23. O'Brien SG, Guilhot F, Larson RA, Gathmann I, Baccarani M, Cervantes F, Cornelissen JJ, Fischer T, Hochhaus A, Hughes T, et al. Imatinib compared with interferon and low-dose cytarabine for newly diagnosed chronic-phase chronic myeloid leukemia. N Engl J Med. 2003;348(11):994-1004.

24. Cortes JE, Baccarani M, Guilhot F, Druker BJ, Branford S, Kim DW, Pane F, Pasquini R, Goldberg SL, Kalaycio M, et al. Phase III, randomized, open-label study of daily imatinib mesylate $400 \mathrm{mg}$ versus $800 \mathrm{mg}$ in patients with newly diagnosed, previously untreated chronic myeloid leukemia in chronic 
phase using molecular end points: tyrosine kinase inhibitor optimization and selectivity study. J Clin Oncol. 2010;28(3):424-30.

25. Baccarani M, Rosti G, Castagnetti F, Haznedaroglu I, Porkka K, Abruzzese E, Alimena $\mathrm{G}$, Ehrencrona $\mathrm{H}$, Hjorth-Hansen $\mathrm{H}$, Kairisto V, et al. Comparison of imatinib $400 \mathrm{mg}$ and $800 \mathrm{mg}$ daily in the front-line treatment of high-risk, Philadelphia-positive chronic myeloid leukemia: a European LeukemiaNet study. Blood. 2009;113(19):4497-504.

26. Preudhomme C, Guilhot J, Nicolini FE, Guerci-Bresler A, Rigal-Huguet F, Maloisel F, Coiteux V, Gardembas M, Berthou C, Vekhoff A, et al. Imatinib plus peginterferon alfa-2a in chronic myeloid leukemia. N Engl J Med. 2010; 363(26):2511-21.

27. Larson RA, Hochhaus A, Hughes TP, Clark RE, Etienne G, Kim DW, Flinn IW, Kurokawa M, Moiraghi B, Yu R, et al. Nilotinib vs imatinib in patients with newly diagnosed Philadelphia chromosome-positive chronic myeloid leukemia in chronic phase: ENESTnd 3-year follow-up. Leukemia. 2012 26(10):2197-203.

28. Saglio G, Kim DW, Issaragrisil S, le Coutre P, Etienne G, Lobo C, Pasquini R, Clark RE, Hochhaus A, Hughes TP, et al. Nilotinib versus imatinib for newly diagnosed chronic myeloid leukemia. N Engl J Med. 2010;362(24):2251-9.

29. Kantarjian HM, Hochhaus A, Saglio G, De Souza C, Flinn IW, Stenke L, Goh YT, Rosti G, Nakamae H, Gallagher NJ, et al. Nilotinib versus imatinib for the treatment of patients with newly diagnosed chronic phase, Philadelphia chromosome-positive, chronic myeloid leukaemia: 24-month minimum follow-up of the phase 3 randomised ENESTnd trial. The Lancet Oncol. 2011; 12(9):841-51.

30. Hochhaus A, Saglio G, Hughes TP, Larson RA, Kim DW, Issaragrisil S, le Coutre PD, Etienne G, Dorlhiac-Llacer PE, Clark RE, et al. Long-term benefits and risks of frontline nilotinib vs imatinib for chronic myeloid leukemia in chronic phase: 5-year update of the randomized ENESTnd trial. Leukemia. 2016;30(5):1044-54.

31. Fujisawa $S$, Nakamae H, Ogura M, Ishizawa K, Taniwaki M, Utsunomiya A, Matsue K, Takamatsu Y, Usuki K, Tanimoto M, et al. Efficacy and safety of dasatinib versus imatinib in Japanese patients with newly diagnosed chronic-phase chronic myeloid leukemia (CML-CP): subset analysis of the DASISION trial with 2-year follow-up. Int J Hematol. 2014;99(2):141-53.

32. Kantarjian H, Shah NP, Hochhaus A, Cortes J, Shah S, Ayala M, Moiraghi B, Shen Z, Mayer J, Pasquini R, et al. Dasatinib versus imatinib in newly diagnosed chronic-phase chronic myeloid leukemia. N Engl J Med. 2010; 362(24):2260-70

33. Jabbour E, Kantarjian HM, Saglio G, Steegmann JL, Shah NP, Boque C, Chuah C, Pavlovsky C, Mayer J, Cortes J, et al. Early response with dasatinib or imatinib in chronic myeloid leukemia: 3-year follow-up from a randomized phase 3 trial (DASISION). Blood. 2014;123(4):494-500.

34. Hughes TP, Saglio G, Quintas-Cardama A, Mauro MJ, Kim DW, Lipton JH, Bradley-Garelik MB, Ukropec J, Hochhaus A. BCR-ABL1 mutation development during first-line treatment with dasatinib or imatinib for chronic myeloid leukemia in chronic phase. Leukemia. 2015;29(9):1832-8.

35. Nakamae H, Fujisawa S, Ogura M, Uchida T, Onishi Y, Taniwaki M, Utsunomiya A, Matsue K, Takamatsu Y, Usuki K, et al. Dasatinib versus imatinib in Japanese patients with newly diagnosed chronic phase chronic myeloid leukemia: a subanalysis of the DASISION 5-year final report. Int J Hematol. 2017;105(6):792-804.

36. Petzer AL, Wolf D, Fong D, Lion T, Dyagil I, Masliak Z, Bogdanovic A, Griskevicius L, Lejniece S, Goranov S, et al. High-dose imatinib improves cytogenetic and molecular remissions in patients with pretreated Philadelphia-positive, BCR-ABL-positive chronic phase chronic myeloid leukemia: first results from the randomized CELSG phase III CML 11 "ISTAHIT" study. Haematologica. 2010;95(6):908-13.

37. Cortes JE, Kim DW, Kantarjian HM, Brummendorf TH, Dyagil I, Griskevicius L, Malhotra H, Powell C, Gogat K, Countouriotis AM, et al. Bosutinib versus imatinib in newly diagnosed chronic-phase chronic myeloid leukemia: results from the BELA trial. J Clin Oncol. 2012;30(28):3486-92.

38. Brummendorf TH, Cortes JE, de Souza CA, Guilhot F, Duvillie L, Pavlov D, Gogat K, Countouriotis AM, Gambacorti-Passerini C. Bosutinib versus imatinib in newly diagnosed chronic-phase chronic myeloid leukaemia: results from the 24-month follow-up of the BELA trial. Br J Haematol. 2015; 168(1):69-81.

39. Gambacorti-Passerini C, Cortes JE, Lipton JH, Dmoszynska A, Wong RS, Rossiev V, Pavlov D, Gogat Marchant K, Duvillie L, Khattry N, et al. Safety of bosutinib versus imatinib in the phase 3 BELA trial in newly diagnosed chronic phase chronic myeloid leukemia. Am J Hematol. 2014;89(10):947-53.
40. Hehlmann R, Lauseker M, Jung-Munkwitz S, Leitner A, Muller MC, Pletsch N, Proetel U, Haferlach C, Schlegelberger B, Balleisen L, et al. Tolerabilityadapted imatinib $800 \mathrm{mg} / \mathrm{d}$ versus $400 \mathrm{mg} / \mathrm{d}$ versus $400 \mathrm{mg} / \mathrm{d}$ plus interferon-alpha in newly diagnosed chronic myeloid leukemia. J Clin Oncol. 2011;29(12):1634-42.

41. Radich JP, Kopecky K, Appelbaum FR, Kamel-Reid S, Stock W, Malnassy G, Paietta E, Wadleigh M, Larson RA, Emanuel P, et al. A randomized trial of dasatinib 100 mg versus imatinib 400 mg in newly diagnosed chronicphase chronic myeloid leukemia. Blood. 2012;120(19):3898-905.

42. Thielen $N$, van der Holt B, Verhoef GE, Ammerlaan RA, Sonneveld P, Janssen $J$ J, Deenik W, Falkenburg JH, Kersten MJ, Sinnige HA, et al. High-dose imatinib versus high-dose imatinib in combination with intermediate-dose cytarabine in patients with first chronic phase myeloid leukemia: a randomized phase III trial of the Dutch-Belgian HOVON study group. Ann Hematol. 2013;92(8):1049-56.

43. Hughes TP, Lipton JH, Spector N, Cervantes F, Pasquini R, Clementino NC, Dorlhiac Llacer PE, Schwarer AP, Mahon FX, Rea D, et al. Deep molecular responses achieved in patients with CML-CP who are switched to nilotinib after long-term imatinib. Blood. 2014;124(5):729-36.

44. O'Brien SG, Hedgley C, Adams S, Foroni L, Apperley JF, Holyoake TL, Pocock C, Byrne JL, Seeley LM, Osborne WL. Spirit 2: an NCRI randomised study comparing Dasatinib with Imatinib in patients with newly diagnosed CML. Blood. 2014;124:517.

45. Osborne DW, O'Brien S, Hedgley C, Bescoby R. SPIRIT 2: an NCRI randomised study comparing dasatinib with imatinib in patients with newly diagnosed chronic myeloid leukaemia-2 year follow up. British Society for Haematology Annual Scientific Meeting Abstract. 2015:15-5. https://doi. org/10.1111/bjh.13350.

46. Deininger MW, Kopecky KJ, Radich JP, Kamel-Reid S, Stock W, Paietta E, Emanuel PD, Tallman M, Wadleigh M, Larson RA, et al. Imatinib 800 mg daily induces deeper molecular responses than imatinib 400 mg daily: results of SWOG S0325, an intergroup randomized PHASE II trial in newly diagnosed chronic phase chronic myeloid leukaemia. Br J Haematol. 2014;164(2):223-32.

47. Hjorth-Hansen H, Stenke L, Soderlund S, Dreimane A, Ehrencrona H, GeddeDahl T, Gjertsen BT, Hoglund M, Koskenvesa P, Lotfi K, et al. Dasatinib induces fast and deep responses in newly diagnosed chronic myeloid leukaemia patients in chronic phase: clinical results from a randomised phase-2 study (NordCML006). Eur J Haematol. 2015;94(3):243-50.

48. Wang J, Shen ZX, Saglio G, Jin J, Huang H, Hu Y, Du X, Li J, Meng F, Zhu H, et al. Phase 3 study of nilotinib vs imatinib in Chinese patients with newly diagnosed chronic myeloid leukemia in chronic phase: ENESTchina. Blood. 2015:125(18):2771-8.

49. Kwak JY, Kim SH, Oh SJ, Zang DY, Kim H, Kim JA, Do YR, Kim HJ, Park JS, Choi CW, et al. Phase III clinical trial (RERISE study) results of efficacy and safety of Radotinib compared with Imatinib in newly diagnosed chronic phase chronic myeloid leukemia. Clin Cancer Res. 2017;23(23):7180-8.

50. Lipton JH, Chuah C, Guerci-Bresler A, Rosti G, Simpson D, Assouline S, Etienne G, Nicolini FE, le Coutre P, Clark RE, et al. Ponatinib versus imatinib for newly diagnosed chronic myeloid leukaemia: an international, randomised, open-label, phase 3 trial. The Lancet Oncol. 2016;17(5):612-21.

51. Cortes JE, De Souza CA, Ayala M, Lopez JL, Bullorsky E, Shah S, Huang X, Babu KG, Abdulkadyrov K, de Oliveira JSR, et al. Switching to nilotinib versus imatinib dose escalation in patients with chronic myeloid leukaemia in chronic phase with suboptimal response to imatinib (LASOR): a randomised, open-label trial. The Lancet Haematol. 2016;3(12):e581-91.

52. Hehlmann R, Lauseker M, Saussele S, Pfirrmann M, Krause S, Kolb HJ, Neubauer A, Hossfeld DK, Nerl C, Gratwohl A, et al. Assessment of imatinib as first-line treatment of chronic myeloid leukemia: 10-year survival results of the randomized CML study IV and impact of non-CML determinants. Leukemia. 2017;31(11):2398-406.

53. Kalmanti L, Saussele $S$, Lauseker M, Muller MC, Dietz CT, Heinrich L, Hanfstein B, Proetel U, Fabarius A, Krause SW, et al. Safety and efficacy of imatinib in CML over a period of 10 years: data from the randomized CMLstudy IV. Leukemia. 2015;29(5):1123-32.

54. Cortes JE, Gambacorti-Passerini C, Deininger MW, Mauro MJ, Chuah C, Kim DW, Dyagil I, Glushko N, Milojkovic D, le Coutre P, et al. Bosutinib versus Imatinib for newly diagnosed chronic myeloid leukemia: results from the randomized BFORE trial. J Clin Oncol. 2018;36(3):231-7.

55. Hughes TP, Hochhaus A, Branford S, Muller MC, Kaeda JS, Foroni L, Druker BJ, Guilhot F, Larson RA, O'Brien SG, et al. Long-term prognostic significance 
of early molecular response to imatinib in newly diagnosed chronic myeloid leukemia: an analysis from the international randomized study of interferon and STI571 (IRIS). Blood. 2010;116(19):3758-65.

56. Jabbour E, Kantarjian H, O'Brien S, Shan J, Quintas-Cardama A, Faderl S, Garcia-Manero G, Ravandi F, Rios MB, Cortes J. The achievement of an early complete cytogenetic response is a major determinant for outcome in patients with early chronic phase chronic myeloid leukemia treated with tyrosine kinase inhibitors. Blood. 2011;118(17):4541-6 quiz 4759.

57. Marin D, Milojkovic D, Olavarria E, Khorashad JS, de Lavallade H, Reid AG, Foroni L, Rezvani K, Bua M, Dazzi F, et al. European LeukemiaNet criteria for failure or suboptimal response reliably identify patients with CML in early chronic phase treated with imatinib whose eventual outcome is poor. Blood. 2008;112(12):4437-44.

58. Giles FJ, Mauro MJ, Hong F, Ortmann CE, McNeill C, Woodman RC, Hochhaus A, le Coutre PD, Saglio G. Rates of peripheral arterial occlusive disease in patients with chronic myeloid leukemia in the chronic phase treated with imatinib, nilotinib, or non-tyrosine kinase therapy: a retrospective cohort analysis. Leukemia. 2013;27(6):1310-5.

59. Pavlovsky C, Chan O, Talati C, Pinilla-lbarz J. Ponatinib in the treatment of chronic myeloid leukemia and philadelphia chromosome positive acute lymphoblastic leukemia. Future Oncol (London, England). 2018;15(3):257-69.

\section{Publisher's Note}

Springer Nature remains neutral with regard to jurisdictional claims in published maps and institutional affiliations.

Ready to submit your research? Choose BMC and benefit from:

- fast, convenient online submission

- thorough peer review by experienced researchers in your field

- rapid publication on acceptance

- support for research data, including large and complex data types

- gold Open Access which fosters wider collaboration and increased citations

- maximum visibility for your research: over $100 \mathrm{M}$ website views per year

At $\mathrm{BMC}$, research is always in progress.

Learn more biomedcentral.com/submissions 